\title{
Research Article \\ Effect of Couple Stresses on the Stress Intensity Factors for Two Parallel Cracks in an Infinite Elastic Medium under Tension
}

\author{
Shouetsu Itou \\ Department of Mechanical Engineering, Kanagawa University, Rokkakubashi, Kanagawa-ku, \\ Yokohama 221-8686, Japan \\ Correspondence should be addressed to Shouetsu Itou, itous001@kanagawa-u.ac.jp
}

Received 8 February 2012; Accepted 22 March 2012

Academic Editor: Oleg V. Gendelman

Copyright (C) 2012 Shouetsu Itou. This is an open access article distributed under the Creative Commons Attribution License, which permits unrestricted use, distribution, and reproduction in any medium, provided the original work is properly cited.

\begin{abstract}
Stresses around two parallel cracks of equal length in an infinite elastic medium are evaluated based on the linearized couple-stress theory under uniform tension normal to the cracks. Fourier transformations are used to reduce the boundary conditions with respect to the upper crack to dual integral equations. In order to solve these equations, the differences in the displacements and in the rotation at the upper crack are expanded through a series of functions that are zero valued outside the crack. The unknown coefficients in each series are solved in order to satisfy the boundary conditions inside the crack using the Schmidt method. The stresses are expressed in terms of infinite integrals, and the stress intensity factors can be determined using the characteristics of the integrands for an infinite value of the variable of integration. Numerical calculations are carried out for selected crack configurations, and the effect of the couple stresses on the stress intensity factors is revealed.
\end{abstract}

\section{Introduction}

In the classical theory of elasticity, the differential equations of equilibrium are derived from the equilibrium of the forces for the rectangular parallelepiped element $d x d y d z$ with respect to the rectangular coordinates $(x, y, z)$. Since the element $d x d y d z$ is infinitesimal, the normal stresses and shearing stresses are considered to act only on the surfaces of the element. The classical theory of elasticity is valid for a homogeneous material. In contrast, for materials with microstructures, such as porous materials and discrete materials, the differential equations of equilibrium may be derived from a parallelepiped element, which, although very small, is not infinitesimal. This produces additional stresses, called couple stresses, on the surfaces of the parallelepiped element. In the linearized couple stress theory 
(also referred to as the Cosserat theory with constrained rotations), the couple stresses are assumed to be proportional to the curvature, which yields a new material constant $l$, the dimension of which is length [1].

Mindlin evaluated the effect of couple stresses on the stress concentration around a circular hole in an infinite medium under tension [1], and Itou examined the effect of the couple stresses on the stress concentration around a circular hole in an infinite elastic layer under tension [2]. A similar problem has been solved for an infinite medium containing an infinite row of equally spaced holes of equal diameter under tension in the linearized couplestress theory [3]. In these studies [1-3], the values of the stress concentration are shown to approach those for the corresponding classical solutions as $l / r$ approaches zero, where $2 r$ is the diameter of the holes.

Sternberg and Muki solved the stress intensity factor around a finite crack in an infinite Cosserat medium under tension and revealed that the Mode I stress intensity factor is always larger than the corresponding value for the classical theory of elasticity [4]. Yoffe assumed that a crack propagates only to the right, maintaining its length $2 a$ to be constant and solved the stress intensity factor for the propagating crack [5]. For the Yoffe model, Itou evaluated the stress intensity factor in the linearized couple-stress theory [6]. For the crack problems, it has been shown that the values of the stress intensity factors are always larger than those for the classical theory of elasticity and that the values increase as $l / a$ approaches zero.

Savin et al. determined the characteristic length $l$ by measuring the velocity of the transverse ultrasonic wave for brass, bronze, duralumin, and aluminum [7], and the material constant $l$ has been shown to be approximately one order of magnitude smaller than the mean grain size [7]. Thus, the effect of the couple stresses does not significantly affect the stress concentrations caused by the existence of circular holes, circular inclusions, and notches, whereas the effect of the couple stresses on the stress intensity factor around a crack is always larger than the corresponding value in the classical theory of elasticity.

If the weight of airplanes, high-speed trains, and automobiles can be reduced, fuel consumption can be curtailed considerably. This may be accomplished by using polycarbonate honeycomb materials and metal foam materials when designing machine elements. Mora and Waas performed a compression test of honeycomb materials with a rigid circular inclusion and estimated that $l / d$ falls in the range 10.0 to 15.0 , where $d$ is the diameter of each cell of the honeycomb [8]. Although no experiment has been performed to determine the value of the characteristic length $l$ for metal foam materials, $l$ may be expected to have a value on the order of the mean average value of the diameter of the foam. The metal foam materials and the polycarbonate honeycomb materials reduce the need for landfills because these materials are reusable. As such, these materials are increasingly being used for structural components in airplanes, high-speed trains, and automobiles. As a result, the couplestress theory has been used increasingly for the evaluation of the stresses produced in such materials.

Gourgiotis and Georgiadis solved the Mode II and Mode III stress intensity factors for a crack in an infinite medium using the couple-stress theory and the distributed dislocation technique [9]. A Mode I crack problem was later solved by Gourgiotis and Georgiadis for a crack in an infinite medium [10]. Recently, Gourgiotis and Georgiadis evaluated the stress field in the vicinity of a sharp notch in an infinite medium under tension and searing stress using the couple-stress theory [11]. To the author's knowledge, the stress intensity factors have been only evaluated for a crack in an infinite medium. In the present paper, stresses are solved for two equal parallel cracks in an infinite medium under tension using the couplestress theory. The Fourier transform technique is used to reduce the boundary conditions with 


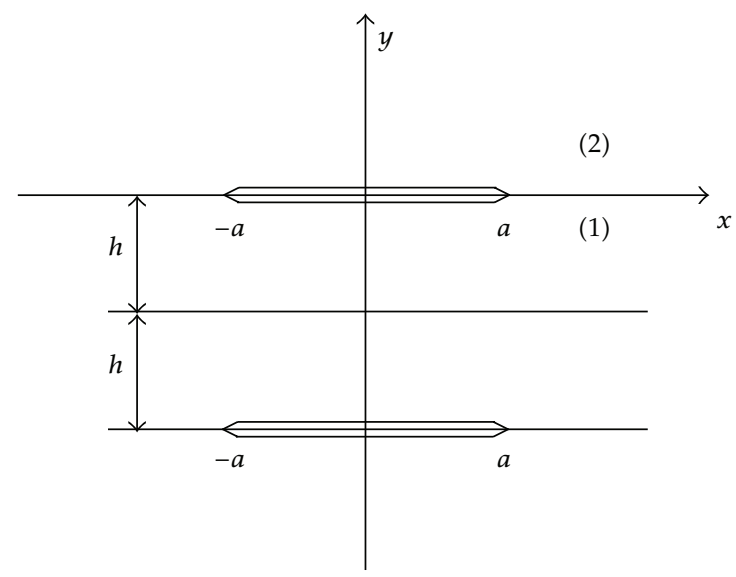

Figure 1: Geometry and coordinate system.

respect to the upper crack to dual integral equations. The differences in the displacements and in the rotation at the upper crack are expanded through a series of functions that vanish outside the crack. The unknown coefficients in each series are solved using the Schmidt method [12]. The stress intensity factors and the couple-stress intensity factor are calculated numerically for several crack configurations.

\section{Fundamental Equations}

In Cartesian coordinates $(x, y)$, the upper crack is located between $-a$ and $a$ at $y=0$, and the lower crack is located between $-a$ and $a$ at $y=-2 h$, as shown in Figure 1. Under plane strain conditions, the force stresses $\tau_{x x}, \tau_{y y}, \tau_{x y}, \tau_{y x}$ and the couple stresses $\mu_{x}, \mu_{y}$ are expressed as follows:

$$
\begin{aligned}
\tau_{x x}=\frac{\partial^{2} \phi}{\partial y^{2}}-\frac{\partial^{2} \psi}{\partial x \partial y}, & \tau_{y y}=\frac{\partial^{2} \phi}{\partial x^{2}}+\frac{\partial^{2} \psi}{\partial x \partial y} \\
\tau_{x y}=-\frac{\partial^{2} \phi}{\partial x \partial y}-\frac{\partial^{2} \psi}{\partial y^{2}}, & \tau_{y x}=-\frac{\partial^{2} \phi}{\partial x \partial y}+\frac{\partial^{2} \psi}{\partial x^{2}} \\
\mu_{x}=\frac{\partial \psi}{\partial x}, & \mu_{y}=\frac{\partial \psi}{\partial y},
\end{aligned}
$$

where $\phi$ and $\psi$ satisfy the following equations:

$$
\begin{gathered}
\nabla^{4} \phi=0, \quad \nabla^{2} \psi-l^{2} \nabla^{4} \psi=0, \\
\frac{\partial}{\partial x}\left(\psi-l^{2} \nabla^{2} \psi\right)=-2(1-v) l^{2} \frac{\partial}{\partial y} \nabla^{2} \phi,
\end{gathered}
$$


where $\nabla^{2}$ is the Laplacian operator, and $l$ is the new material constant. The rotation $\omega_{z}$ and the strains $\varepsilon_{x}$ and $\varepsilon_{y}$ are given as follows:

$$
\begin{gathered}
\omega_{z}=\frac{1}{2} \times\left(\frac{\partial v}{\partial x}-\frac{\partial u}{\partial y}\right) \\
2 G \varepsilon_{y}=2 G \frac{\partial v}{\partial y}=(1-v) \tau_{y y}-v \tau_{x x}, \quad 2 G \varepsilon_{x}=2 G \frac{\partial u}{\partial x}=(1-v) \tau_{x x}-v \tau_{y y}
\end{gathered}
$$

where $u$ and $v$ are the $x$ and $y$ components of the displacement, and $G$ and $v$ are the shear modulus and Poisson's ratio, respectively.

\section{Boundary Conditions}

If we assume that a tensile stress $p$ is applied perpendicular to the two cracks, the stress field is symmetric with respect to the plane $y=-h$, and it is sufficient to solve the problem for $-h<y<\infty$ only. For convenience, we refer to the layer $-h<y<0$ as layer (1) and to the upper half-plane $0<y<\infty$ as half plane (2). The boundary conditions can be expressed as follows:

$$
\begin{gathered}
\tau_{y y 1}^{0}=-p, \quad \text { for }|x|<a, y=0, \\
\tau_{y x 1}^{0}=0, \quad \text { for }|x|<a, y=0, \\
\mu_{y 1}^{0}=0, \quad \text { for }|x|<a, y=0, \\
u_{1}^{0}=u_{2}^{0}, \quad \text { for } a<|x|, y=0, \\
v_{1}^{0}=v_{2}^{0}, \quad \text { for } a<|x|, y=0, \\
\omega_{z 1}^{0}=\omega_{z 2}^{0}, \quad \text { for } a<|x|, y=0, \\
\tau_{y y 1}^{0}=\tau_{y y 2}^{0}, \quad \text { for }|x|<\infty, y=0, \\
\tau_{y x 1}^{0}=\tau_{y x 2}^{0}, \quad \text { for }|x|<\infty, y=0, \\
\mu_{y 1}^{0}=\mu_{y 2}^{0}, \quad \text { for }|x|<\infty, y=0, \\
\tau_{y x 1}^{-h}=0, \quad \text { for }|x|<\infty, y=-h, \\
v_{1}^{-h}=0, \quad \text { for }|x|<\infty, y=-h, \\
\omega_{z 1}^{-h}=0, \quad \text { for }|x|<\infty, y=-h,
\end{gathered}
$$

where subscripts 1 and 2 indicate layer (1) and half plane (2), respectively, and superscripts 0 and $-h$ indicate the values at $y=0$ and $y=-h$, respectively. 


\section{Analysis}

In order to find the solution, the Fourier transforms are introduced as follows:

$$
\begin{gathered}
\bar{f}(\xi)=\int_{-\infty}^{\infty} f(x) \exp (i \xi x) d x \\
f(x)=\frac{1}{2 \pi} \int_{-\infty}^{\infty} \bar{f}(\xi) \exp (-i \xi x) d \xi .
\end{gathered}
$$

Applying (4.1) to (2.2) yields the following:

$$
\begin{gathered}
\frac{d^{4} \bar{\phi}}{d y^{4}}-2 \xi^{2} \frac{d^{2} \bar{\phi}}{d y^{2}}+\xi^{4} \bar{\phi}=0 \\
l^{2} \frac{d^{4} \bar{\psi}}{d y^{4}}-\left(2 \xi^{2} l^{2}+1\right) \frac{d^{2} \bar{\psi}}{d y^{2}}+\xi^{2}\left(\xi^{2} l^{2}+1\right) \bar{\psi}=0 .
\end{gathered}
$$

The solutions for (4.3) take the following forms for $i=1$ and 2 :

$$
\begin{gathered}
\bar{\phi}_{1}=A_{1} \cosh (\xi y)+B_{1} y \cosh (\xi y)+C_{1} \sinh (\xi y)+D_{1} y \sinh (\xi y) \\
\bar{\psi}_{1}=E_{1} \cosh (k y)+F_{1} \cosh (\xi y)+H_{1} \sinh (k y)+I_{1} \sinh (\xi y), \\
\bar{\phi}_{2}=\left(A_{2}+B_{2} y\right) \exp (-|\xi| y), \\
\bar{\psi}_{2}=E_{2} \exp (-|\xi| y)+F_{2} \exp (-k y),
\end{gathered}
$$

where $A_{1}, B_{1}, C_{1}, D_{1}, E_{1}, F_{1}, H_{1}, I_{1}, A_{2}, B_{2}, E_{2}$, and $F_{2}$ are unknown coefficients, and $k$ is given by:

$$
k=\sqrt{\frac{\left(\xi^{2} l^{2}+1\right)}{l^{2}}} .
$$

Using the Fourier transformed expressions of (2.3), the coefficients $I_{1}, F_{1}$, and $E_{2}$ can be represented by the coefficients $D_{1}, B_{1}$, and $B_{2}$ as follows:

$$
I_{1}=-4 i(1-v) l^{2} \xi D_{1}, \quad F_{1}=-4 i(1-v) l^{2} \xi B_{1}, \quad E_{2}=-4 i(1-v) l^{2} \xi B_{2} .
$$

Thus, the stresses, the displacements, and the rotation can be expressed by nine unknown coefficients in the Fourier domain.

Using (3.10) through (3.12), which are valid for $-\infty<x<+\infty$, unknowns $C_{1}, D_{1}$, and $E_{1}$ are given as follows:

$$
\begin{gathered}
C_{1}=A_{1} c_{11}+B_{1} c_{12}+i H_{1} c_{13}, \quad D_{1}=A_{1} c_{21}+B_{1} c_{22}+i H_{1} c_{23} \\
i E_{1}=A_{1} c_{31}+B_{1} c_{32}+i H_{1} c_{33}
\end{gathered}
$$


where the expressions of the known functions $c_{i j}(i, j=1,2,3)$ are omitted. Then, the Fouriertransformed expressions of the stresses, the displacements, and the rotation in layer (1) can be expressed in terms of only three unknown coefficients, that are, $A_{1}, B_{1}$, and $H_{1}$. Thus, the displacements $2 G \bar{u}_{1}^{0}$ and $2 G \bar{v}_{1}^{0}$ at $y=0$ and the rotation $4 G \bar{\omega}_{z 1}^{0}$ at $y=0$ can be expressed in terms of three unknown coefficients, that are, $A_{1}, B_{1}$, and $H_{1}$. In contrast, coefficients $A_{1}, B_{1}$, and $H_{1}$ can be expressed as $2 G \bar{u}_{1}^{0}, 2 G \bar{v}_{1}^{0}$, and $4 G \bar{\omega}_{z 1}^{0}$, and then the stresses, the displacements, and the rotation can be expressed in terms of $2 G \bar{u}_{1}^{0}, 2 G \bar{v}_{1}^{0}$, and $4 G \bar{\omega}_{z 1}^{0}$ in the Fourier domain. For examples, $\bar{\tau}_{y y 1}^{0}, \bar{\tau}_{y x 1}^{0}$, and $\bar{\mu}_{y 1}^{0}$ have the following forms:

$$
\begin{aligned}
& \bar{\tau}_{y y 1}^{0}=\left(-i \bar{u}_{1}^{0}\right) k_{1}^{1}(\xi)+\bar{v}_{1}^{0} k_{2}^{1}(\xi)+\left(-i \bar{\omega}_{z 1}^{0}\right) k_{3}^{1}(\xi), \\
& \bar{\tau}_{y x 1}^{0}=\left(-i \bar{u}_{1}^{0}\right) i k_{4}^{1}(\xi)+\bar{v}_{1}^{0} i k_{5}^{1}(\xi)+\left(-i \bar{\omega}_{z 1}^{0}\right) i k_{6}^{1}(\xi), \\
& \bar{\mu}_{y 1}^{0}=\left(-i \bar{u}_{1}^{0}\right) i k_{7}^{1}(\xi)+\bar{v}_{1}^{0} i k_{8}^{1}(\xi)+\left(-i \bar{\omega}_{z 1}^{0}\right) i k_{9}^{1}(\xi),
\end{aligned}
$$

where the expressions of the known functions $k_{i}^{1}(\xi)(i=1,2,3, \ldots, 9)$ are omitted.

As for the upper half plane (2), the stresses, the displacements, and the rotation are shown by the three unknown coefficients $A_{2}, B_{2}$, and $F_{2}$. Thus, the displacements $2 G \bar{u}_{2}^{0}$ and $2 G \bar{v}_{2}^{0}$ at $y=0$ and the rotation $4 G \bar{\omega}_{z 2}^{0}$ at $y=0$ can be described by three unknown coefficients $A_{2}, B_{2}$, and $F_{2}$. In a similar manner to the case for layer (1), the unknown coefficients $A_{2}, B_{2}$, and $F_{2}$ are represented by $2 G \bar{u}_{2}^{0}, 2 G \bar{v}_{2}^{0}$, and $4 G \bar{\omega}_{z 2}^{0}$. Then, the stresses, the displacements, and the rotation can be expressed in terms of $2 G \bar{u}_{2}^{0}, 2 G \bar{v}_{2}^{0}$, and $4 G \bar{\omega}_{z 2}^{0}$ in the Fourier domain. For examples, $\bar{\tau}_{y y 2}^{0}, \bar{\tau}_{y x 2}^{0}$, and $\bar{\mu}_{y 2}^{0}$ have following forms:

$$
\begin{aligned}
& \bar{\tau}_{y y 2}^{0}=\left(-i \bar{u}_{2}^{0}\right) k_{1}^{2}(\xi)+\bar{v}_{2}^{0} k_{2}^{2}(\xi)+\left(-i \bar{\omega}_{z 2}^{0}\right) k_{3}^{2}(\xi), \\
& \bar{\tau}_{y x 2}^{0}=\left(-i \bar{u}_{2}^{0}\right) i k_{4}^{2}(\xi)+\bar{v}_{2}^{0} i k_{5}^{2}(\xi)+\left(-i \bar{\omega}_{z 2}^{0}\right) i k_{6}^{2}(\xi), \\
& \bar{\mu}_{y 2}^{0}=\left(-i \bar{u}_{2}^{0}\right) i k_{7}^{2}(\xi)+\bar{v}_{2}^{0} i k_{8}^{2}(\xi)+\left(-i \bar{\omega}_{z 2}^{0}\right) i k_{9}^{2}(\xi),
\end{aligned}
$$

where the expressions of the known functions $k_{i}^{2}(\xi)(i=1,2,3, \ldots, 9)$ are omitted.

Using (3.7), (3.8) and (3.9) the following relations are obtained:

$$
\begin{gathered}
\left(-i \bar{u}_{1}^{0}\right) k_{1}^{1}(\xi)+\bar{v}_{1}^{0} k_{2}^{1}(\xi)+\left(-i \bar{\omega}_{z 1}^{0}\right) k_{3}^{1}(\xi) \\
=\left(-i \bar{u}_{2}^{0}\right) k_{1}^{2}(\xi)+\bar{v}_{2}^{0} k_{2}^{2}(\xi)+\left(-i \bar{\omega}_{z 2}^{0}\right) k_{3}^{2}(\xi), \\
\left(-i \bar{u}_{1}^{0}\right) i k_{4}^{1}(\xi)+\bar{\nu}_{1}^{0} i k_{5}^{1}(\xi)+\left(-i \bar{\omega}_{z 1}^{0}\right) i k_{6}^{1}(\xi) \\
=\left(-i \bar{u}_{2}^{0}\right) i k_{4}^{2}(\xi)+\bar{\nu}_{2}^{0} i k_{5}^{2}(\xi)+\left(-i \bar{\omega}_{z 2}^{0}\right) i k_{6}^{2}(\xi), \\
\left(-i \bar{u}_{1}^{0}\right) i k_{7}^{1}(\xi)+\bar{v}_{1}^{0} i k_{8}^{1}(\xi)+\left(-i \bar{\omega}_{z 1}^{0}\right) i k_{9}^{1}(\xi) \\
=\left(-i \bar{u}_{2}^{0}\right) i k_{7}^{2}(\xi)+\bar{v}_{2}^{0} i k_{8}^{2}(\xi)+\left(-i \bar{\omega}_{z 2}^{0}\right) i k_{9}^{2}(\xi) .
\end{gathered}
$$


Equation (4.10) can be solved for $\left(-i \bar{u}_{1}^{0}\right), \bar{v}_{1}^{0}$ and $\left(-i \bar{\omega}_{z 1}^{0}\right)$ as follows:

$$
\begin{gathered}
\left(-i \bar{u}_{1}^{0}\right)=\left(-i \bar{u}_{2}^{0}\right) l_{1}(\xi)+\bar{v}_{2}^{0} l_{2}(\xi)+\left(-i \bar{\omega}_{z 2}^{0}\right) l_{3}(\xi), \\
\bar{\nu}_{1}^{0}=\left(-i \bar{u}_{2}^{0}\right) l_{4}(\xi)+\bar{v}_{2}^{0} l_{5}(\xi)+\left(-i \bar{\omega}_{z 2}^{0}\right) l_{6}(\xi), \\
\left(-i \bar{\omega}_{1}^{0}\right)=\left(-i \bar{u}_{2}^{0}\right) l_{7}(\xi)+\bar{\nu}_{2}^{0} l_{8}(\xi)+\left(-i \bar{\omega}_{z 2}^{0}\right) l_{9}(\xi),
\end{gathered}
$$

where the expressions of the known functions $l_{i}(\xi)(i=1,2,3, \ldots, 9)$ are omitted.

In order to satisfy (3.4), (3.5), and (3.6) the differences in the displacements and in the rotation at $y=0$ are expanded by the following series:

$$
\begin{aligned}
\pi\left(v_{1}^{0}-v_{2}^{0}\right) & =\sum_{n=1}^{\infty} c_{n} \cos \left[(2 n-1) \sin ^{-1}\left(\frac{x}{a}\right)\right] \quad \text { for }|x| \leq a \\
& =0 \quad \text { for } a \leq|x| \leq \infty, \\
\pi\left(u_{1}^{0}-u_{2}^{0}\right) & =\sum_{n=1}^{\infty} d_{n} \sin \left[2 n \sin ^{-1}\left(\frac{x}{a}\right)\right] \quad \text { for }|x| \leq a \\
& =0 \quad \text { for } a \leq|x| \leq \infty, \\
\pi\left(\omega_{z 1}^{0}-\omega_{z 2}^{0}\right) & =\sum_{n=1}^{\infty} e_{n} \sin \left[2 n \sin ^{-1}\left(\frac{x}{a}\right)\right] \quad \text { for }|x| \leq a \\
& =0 \text { for } a \leq|x| \leq \infty,
\end{aligned}
$$

where $c_{n}, d_{n}$, and $e_{n}$ are the unknown coefficients that are to be determined. The Fourier transforms of (4.12) through (4.14) are

$$
\begin{aligned}
& \left(\bar{v}_{1}^{0}-\bar{\nu}_{2}^{0}\right)=\sum_{n=1}^{\infty} c_{n} \frac{(2 n-1)}{\xi} J_{2 n-1}(a \xi) \\
& \left(\bar{u}_{1}^{0}-\bar{u}_{2}^{0}\right)=i \sum_{n=1}^{\infty} d_{n} \frac{2 n}{\xi} J_{2 n}(a \xi) \\
& \left(\bar{\omega}_{z 1}^{0}-\bar{\omega}_{z 2}^{0}\right)=i \sum_{n=1}^{\infty} e_{n} \frac{2 n}{\xi} J_{2 n}(a \xi)
\end{aligned}
$$

where $J_{n}(\xi)$ is the Bessel function. 
Substituting (4.11) into (4.15), we obtain the following equations:

$$
\begin{aligned}
& \left(-i \bar{u}_{2}^{0}\right) l_{4}(\xi)+\bar{v}_{2}^{0}\left[l_{5}(\xi)-1\right]+\left(-i \bar{\omega}_{z 2}^{0}\right) l_{6}(\xi)=\sum_{n=1}^{\infty} c_{n} \frac{(2 n-1)}{\xi} J_{2 n-1}(a \xi), \\
& \left(-i \bar{u}_{2}^{0}\right)\left[l_{1}(\xi)-1\right]+\bar{v}_{2}^{0} l_{2}(\xi)+\left(-i \bar{\omega}_{z 2}^{0}\right) l_{3}(\xi)=\sum_{n=1}^{\infty} d_{n} \frac{2 n}{\xi} J_{2 n}(a \xi), \\
& \left(-i \bar{u}_{2}^{0}\right) l_{7}(\xi)+\bar{v}_{2}^{0} l_{8}(\xi)+\left(-i \bar{\omega}_{z 2}^{0}\right)\left[l_{9}(\xi)-1\right]=\sum_{n=1}^{\infty} e_{n} \frac{2 n}{\xi} J_{2 n}(a \xi) .
\end{aligned}
$$

Equations (4.16) can be solved for $\left(-i \bar{u}_{2}^{0}\right), \bar{v}_{2}^{0}$ and $\left(-i \bar{\omega}_{z 2}^{0}\right)$ as follows:

$$
\begin{aligned}
\left(-i \bar{u}_{2}^{0}\right)= & \sum_{n=1}^{\infty} c_{n} \frac{m_{1}(\xi)(2 n-1)}{\xi} J_{2 n-1}(a \xi)+\sum_{n=1}^{\infty} d_{n} \frac{m_{2}(\xi) \times 2 n}{\xi} J_{2 n}(a \xi) \\
& +\sum_{n=1}^{\infty} e_{n} \frac{m_{3}(\xi) \times 2 n}{\xi} J_{2 n}(a \xi), \\
\bar{v}_{2}^{0}= & \sum_{n=1}^{\infty} c_{n} \frac{m_{4}(\xi)(2 n-1)}{\xi} J_{2 n-1}(a \xi)+\sum_{n=1}^{\infty} d_{n} \frac{m_{5}(\xi) \times 2 n}{\xi} J_{2 n}(a \xi) \\
& +\sum_{n=1}^{\infty} e_{n} \frac{m_{6}(\xi) \times 2 n}{\xi} J_{2 n}(a \xi), \\
\left(-i \bar{\omega}_{z 2}^{0}\right)= & \sum_{n=1}^{\infty} c_{n} \frac{m_{7}(\xi)(2 n-1)}{\xi} J_{2 n-1}(a \xi)+\sum_{n=1}^{\infty} d_{n} \frac{m_{8}(\xi) \times 2 n}{\xi} J_{2 n}(a \xi) \\
& +\sum_{n=1}^{\infty} e_{n} \frac{m_{9}(\xi) \times 2 n}{\xi} J_{2 n}(a \xi),
\end{aligned}
$$

where the expressions of the known functions $m_{i}(\xi)(i=1,2,3, \ldots, 9)$ are omitted. At this stage, the stresses, the displacements, and the rotation are represented by only three unknown coefficients, that are, $c_{n}, d_{n}$, and $e_{n}$. For example, stresses $\tau_{y y 2}^{0}, \tau_{y x 2}^{0}$, and $\mu_{y 2}^{0}$ are expressed as follows:

$$
\begin{aligned}
\tau_{y y 2}^{0}= & \sum_{n=1}^{\infty} c_{n} \frac{(2 n-1)}{\pi} \times \int_{0}^{\infty} \frac{q_{1}(\xi)}{\xi} J_{2 n-1}(a \xi) \cos (\xi x) d \xi \\
& +\sum_{n=1}^{\infty} d_{n} \frac{2 n}{\pi} \times \int_{0}^{\infty} \frac{q_{2}(\xi)}{\xi} J_{2 n}(a \xi) \cos (\xi x) d \xi \\
& +\sum_{n=1}^{\infty} e_{n} \frac{2 n}{\pi} \times \int_{0}^{\infty} \frac{q_{3}(\xi)}{\xi} J_{2 n}(a \xi) \cos (\xi x) d \xi
\end{aligned}
$$


Mathematical Problems in Engineering

$$
\begin{aligned}
\tau_{y x 2}^{0}= & \sum_{n=1}^{\infty} c_{n} \frac{(2 n-1)}{\pi} \times \int_{0}^{\infty} \frac{q_{4}(\xi)}{\xi} J_{2 n-1}(a \xi) \sin (\xi x) d \xi \\
& +\sum_{n=1}^{\infty} d_{n} \frac{2 n}{\pi} \times \int_{0}^{\infty} \frac{q_{5}(\xi)}{\xi} J_{2 n}(a \xi) \sin (\xi x) d \xi \\
& +\sum_{n=1}^{\infty} e_{n} \frac{2 n}{\pi} \times \int_{0}^{\infty} \frac{q_{6}(\xi)}{\xi} J_{2 n}(a \xi) \sin (\xi x) d \xi, \\
\mu_{y 2}^{0}= & \sum_{n=1}^{\infty} c_{n} \frac{(2 n-1)}{\pi} \times \int_{0}^{\infty} \frac{q_{7}(\xi)}{\xi} J_{2 n-1}(a \xi) \sin (\xi x) d \xi \\
& +\sum_{n=1}^{\infty} d_{n} \frac{2 n}{\pi} \times \int_{0}^{\infty} \frac{q_{8}(\xi)}{\xi} J_{2 n}(a \xi) \sin (\xi x) d \xi \\
& +\sum_{n=1}^{\infty} e_{n} \frac{2 n}{\pi} \times \int_{0}^{\infty} \frac{q_{9}(\xi)}{\xi} J_{2 n}(a \xi) \sin (\xi x) d \xi,
\end{aligned}
$$

where the known expressions $q_{1}(\xi), q_{2}(\xi), \ldots, q_{8}(\xi)$, and $q_{9}(\xi)$ are omitted. Functions $q_{i}(\xi) /$ $\xi(i=2,3,4,6,7,8)$ decrease rapidly as $\xi$ increases. Functions $q_{i}(\xi) / \xi(i=1,5,9)$ have the following property when $\xi$ increases:

$$
\frac{q_{i}(\xi)}{\xi} \longrightarrow q_{i}^{L}
$$

where constants $q_{i}^{L}(i=1,5,9)$ can be calculated as

$$
q_{i}^{L}=\frac{q_{i}\left(\xi_{L}\right)}{\xi_{L}}
$$

with $\xi_{L}$ being a large value of $\xi$.

Finally, the remaining boundary conditions (3.1), (3.2), and (3.3) can be reduced to the following equations:

$$
\begin{aligned}
& \sum_{n=1}^{\infty} c_{n} K_{n}(x)+\sum_{n=1}^{\infty} d_{n} L_{n}(x)+\sum_{n=1}^{\infty} e_{n} M_{n}(x)=-u(x), \\
& \sum_{n=1}^{\infty} c_{n} O_{n}(x)+\sum_{n=1}^{\infty} d_{n} P_{n}(x)+\sum_{n=1}^{\infty} e_{n} Q_{n}(x)=-v(x), \\
& \sum_{n=1}^{\infty} c_{n} R_{n}(x)+\sum_{n=1}^{\infty} d_{n} S_{n}(x)+\sum_{n=1}^{\infty} e_{n} T_{n}(x)=-w(x) \text { for }|x| \leq a,
\end{aligned}
$$


with

$$
\begin{aligned}
& K_{n}(x)=\frac{(2 n-1)}{\pi} \times\left\{\int_{0}^{\infty}\left[\frac{q_{1}(\xi)}{\xi}-q_{1}^{L}\right] J_{2 n-1}(a \xi) \cos (\xi x) d \xi\right. \\
& \left.+\frac{q_{1}^{L} \cos \left[(2 n-1) \sin ^{-1}(x / a)\right]}{\left(a^{2}-x^{2}\right)^{1 / 2}}\right\} \\
& L_{n}(x)=\frac{2 n}{\pi} \int_{0}^{\infty} \frac{q_{2}(\xi)}{\xi} J_{2 n}(a \xi) \cos (\xi x) d \xi, \\
& M_{n}(x)=\frac{2 n}{\pi} \int_{0}^{\infty} \frac{q_{3}(\xi)}{\xi} J_{2 n}(a \xi) \cos (\xi x) d \xi, \\
& O_{n}(x)=\frac{(2 n-1)}{\pi} \int_{0}^{\infty} \frac{q_{4}(\xi)}{\xi} J_{2 n-1}(a \xi) \sin (\xi x) d \xi, \\
& P_{n}(x)=\frac{2 n}{\pi} \times\left\{\int_{0}^{\infty}\left[\frac{q_{5}(\xi)}{\xi}-q_{5}^{L}\right] J_{2 n}(a \xi) \sin (\xi x) d \xi\right. \\
& \left.+\frac{q_{5}^{L} \sin \left[2 n \sin ^{-1}(x / a)\right]}{\left(a^{2}-x^{2}\right)^{1 / 2}}\right\} \\
& Q_{n}(x)=\frac{2 n}{\pi} \int_{0}^{\infty} \frac{q_{6}(\xi)}{\xi} J_{2 n}(a \xi) \sin (\xi x) d \xi, \\
& R_{n}(x)=\frac{(2 n-1)}{\pi} \int_{0}^{\infty} \frac{q_{7}(\xi)}{\xi} J_{2 n-1}(a \xi) \sin (\xi x) d \xi, \\
& S_{n}(x)=\frac{2 n}{\pi} \int_{0}^{\infty} \frac{q_{8}(\xi)}{\xi} J_{2 n}(a \xi) \sin (\xi x) d \xi, \\
& T_{n}(x)=\frac{2 n}{\pi} \times\left\{\int_{0}^{\infty}\left[\frac{q_{9}(\xi)}{\xi}-q_{9}^{L}\right] J_{2 n}(a \xi) \sin (\xi x) d \xi\right. \\
& \left.+\frac{q_{9}^{L} \sin \left[2 n \sin ^{-1}(x / a)\right]}{\left(a^{2}-x^{2}\right)^{1 / 2}}\right\} \\
& u(x)=p, \quad v(x)=0, \quad w(x)=0
\end{aligned}
$$

The unknown coefficients $c_{n}, d_{n}$, and $e_{n}$ in (4.23) can be solved by the Schmidt method [12]. 


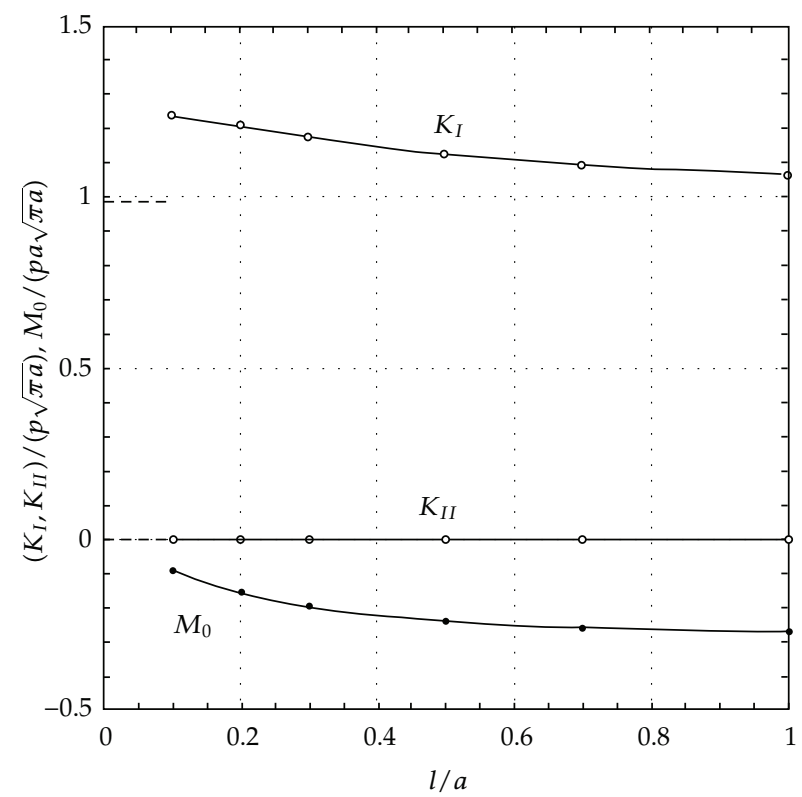

Figure 2: Values of $K_{I}, K_{I I}$ and $M_{0}$ plotted with respect to $l / a$ for $h / a=5.0$ (broken lines show the values for $l / a=0.0$ ).

\section{Stress Intensity Factors}

If we slightly modify the integrands in (4.18) through (4.20) and use the relations

$$
\begin{aligned}
\int_{0}^{\infty} J_{n}(a \xi)[\cos (\xi x), \sin (\xi x)] d \xi & \\
= & \left\{-a^{n}\left(x^{2}-a^{2}\right)^{-1 / 2}\left[x+\left(x^{2}-a^{2}\right)^{-1 / 2}\right]^{-n} \sin \left(\frac{n \pi}{2}\right),\right. \\
& \left.a^{n}\left(x^{2}-a^{2}\right)^{-1 / 2}\left[x+\left(x^{2}-a^{2}\right)^{-1 / 2}\right]^{-n} \cos \left(\frac{n \pi}{2}\right)\right\}, \text { for } a<x,
\end{aligned}
$$

the stress intensity factors and the couple-stress intensity factor can be determined as follows:

$$
\begin{aligned}
& K_{I}=\left.[2 \pi(x-a)]^{1 / 2} \tau_{y y 2}^{0}\right|_{x \rightarrow a+}=\sum_{n=1}^{\infty} c_{n} \frac{(2 n-1)(-1)^{n} q_{1}^{L}}{(\pi a)^{1 / 2}}, \\
& K_{I I}=\left.[2 \pi(x-a)]^{1 / 2} \tau_{y x 2}^{0}\right|_{x \rightarrow a+}=\sum_{n=1}^{\infty} d_{n} \frac{(2 n)(-1)^{n} q_{5}^{L}}{(\pi a)^{1 / 2}}, \\
& M_{0}=\left.[2 \pi(x-a)]^{1 / 2} \mu_{y 2}^{0}\right|_{x \rightarrow a+}=\sum_{n=1}^{\infty} e_{n} \frac{(2 n)(-1)^{n} q_{9}^{L}}{(\pi a)^{1 / 2}} .
\end{aligned}
$$


Table 1: Values of $K_{I} /(p \sqrt{\pi a})$ for $h / a=5.0$. (Values in parentheses were obtained from the diagram in [4]).

\begin{tabular}{lcccc}
\hline$l / a$ & 0.1 & 0.2 & 0.5 & 1.0 \\
\hline$K_{I} /(p \sqrt{\pi a})$ & 1.238 & 1.209 & 1.122 & 1.061 \\
& $(1.231)$ & $(1.202)$ & $(1.120)$ & $(1.063)$ \\
\hline
\end{tabular}

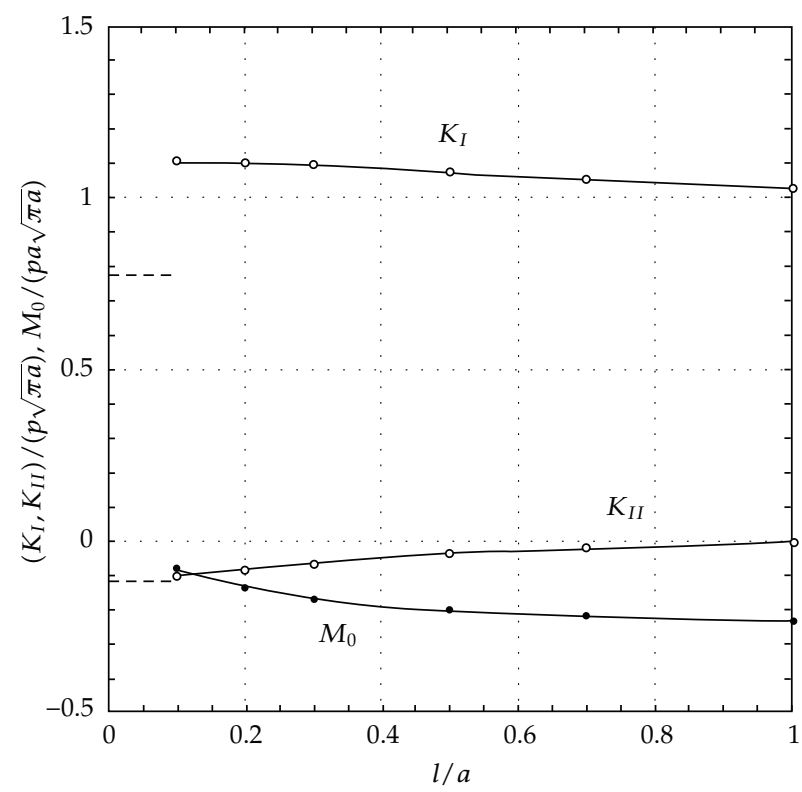

Figure 3: Values of $K_{I}, K_{I I}$, and $M_{0}$ plotted with respect to $l / a$ for $h / a=0.5$ (broken lines show the values for $l / a=0.0$ ).

\section{Numerical Examples}

Numerical calculations are performed with quadruple precision using a Fortran program for which overflow and underflow do not occur within the range of from $10^{-5500}$ to $10^{+5500}$. The stress intensity factors and the couple-stress intensity factor are calculated for $h / a=5.0,0.5$, and 0.1 with a Poisson's ratio of $v=0.25$.

The values of the functions $q_{i}(\xi a) /(\xi a)(i=2,3,4,6,7,8)$ are verified to decay rapidly as $(\xi a)$ increases, and the values of the functions $q_{i}(\xi a) /(\xi a)(i=1,5,9)$ are verified to rapidly approach constants $q_{i}^{L}(i=1,5,9)$ as $(\xi a)$ increases. Thus, the semi-infinite integrals in (4.24) can be evaluated numerically. The Schmidt method, truncated to 12 terms for an infinite series, was then applied to solve for coefficients $c_{n}, d_{n}$, and $e_{n}$ in (4.23). The values of the left-hand side of (4.23) are verified to coincide with those of the right-hand side of (4.23). Then, coefficients $c_{n}, d_{n}$, and $e_{n}$ are verified to be successfully determined by the Schmidt method.

The values of the Mode I stress intensity $K_{I} /(p \sqrt{\pi a})$ are shown for $h / a=5.0$ in Table 1, in which the values in parentheses are obtained from the diagram in [4]. For $h / a=5.0$, the distance between two cracks is $10 a$, and the lower crack is considered not to affect the stress field around the upper crack and vice versa. Both values in Table 1 are well coincident with each other, and the accuracy of the method described in the present paper is 


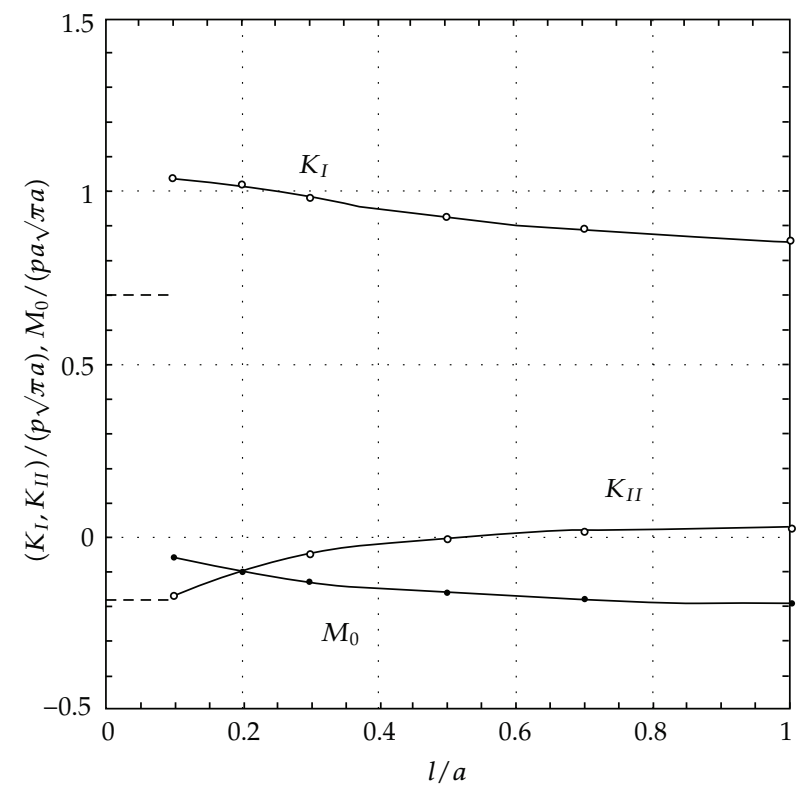

Figure 4: Values of $K_{I}, K_{I I}$, and $M_{0}$ plotted with respect to $l / a$ for $h / a=0.1$ (broken lines show the values for $l / a=0.0)$.

considered to be superior. The values of $K_{I}, K_{I I}$, and $\mathrm{M}_{0}$ are plotted with respect to $l / a$ for $h / a=5.0,0.5$, and 0.1, respectively, in Figures 2, 3, and 4, in which the straight broken lines indicate the corresponding value for $l / a=0.0$.

\section{Conclusion}

Based on the numerical calculations described above, the following conclusions are obtained.

(i) The values of $K_{I} /(p \sqrt{\pi a})$ for $h / a=5.0$ are considered to be approximately coincident with those for a crack in an infinite medium, and the values of $K_{I I} /(p \sqrt{\pi a})$ are considered to be approximately equal to zero.

(ii) As $l / a$ approaches zero, $K_{I} /(p \sqrt{\pi a})$ and $K_{I I} /(p \sqrt{\pi a})$ do not approach the corresponding values calculated using the classical theory of elasticity, whereas the values of $M_{0} /(p a \sqrt{\pi a})$ approach zero, which is the value calculated by the classical theory of elasticity.

(iii) The values of $K_{I} /(p \sqrt{\pi a})$ decrease as $h / a$ decreases, and the same behavior is observed for the absolute values of $M_{0} /(p a \sqrt{\pi a})$.

(iv) The new material constant $l$ may be comparatively small, even for materials with microstructures. Therefore, the key value is $K_{I} /(p \sqrt{\pi a})$, even for materials with microstructures, because the values of $K_{I I} /(p \sqrt{\pi a})$ and $M_{0} /(p a \sqrt{\pi a})$ are considerably smaller than the value of $K_{I} /(p \sqrt{\pi a})$. 


\section{References}

[1] R. D. Mindlin, "Influence of couple-stresses on stress concentrations-main features of Cosserat theory are reviewed by lecturer and some recent solutions of the equations, for cases of stress concentration around small holes in elastic solids, are described," Experimental Mechanics, vol. 3, no. 1, pp. 1-7, 1963.

[2] S. Itou, "The effect of couple-stresses on stress concentrations around a circular hole in a strip under tension," International Journal of Engineering Science, vol. 14, no. 9, pp. 861-867, 1976.

[3] S. Itou, "The effect of couple-stresses on stress concentrations in a plate containing an infinite row of holes," Letters in Applied and Engineering Sciences, vol. 5, pp. 351-358, 1977.

[4] E. Sternberg and R. Muki, "The effect of couple-stresses on the stress concentration around a crack," International Journal of Solids and Structures, vol. 3, no. 1, pp. 69-95, 1967.

[5] E. H. Yoffe, "The moving Griffith crack," Philosophical Magazine, vol. 42, pp. 739-750, 1951.

[6] S. Itou, "The effect of couple-stresses on the stress concentration around a moving crack," International Journal of Mathematics and Mathematical Sciences, vol. 4, no. 1, pp. 165-180, 1981.

[7] G. N. Savin, A. A. Lukasev, E. M. Lysko, S. V. Veremejenko, and G. G. Agasjev, "Elastic wave propagation in a Cosserat continuum with constrained particle rotation," Prikladnaya Mekhanika, vol. 6, pp. 37-41, 1970.

[8] R. Mora and A. M. Waas, "Measurement of the Cosserat constant of circular-cell polycarbonate honeycomb," Philosophical Magazine A, vol. 80, no. 7, pp. 1699-1713, 2000.

[9] P. A. Gourgiotis and H. G. Georgiadis, "Distributed dislocation approach for cracks in couple-stress elasticity: shear modes," International Journal of Fracture, vol. 147, no. 1-4, pp. 83-102, 2007.

[10] P. A. Gourgiotis and H. G. Georgiadis, "An approach based on distributed dislocations and disclinations for crack problems in couple-stress elasticity," International Journal of Solids and Structures, vol. 45, no. 21, pp. 5521-5539, 2008.

[11] P. A. Gourgiotis and H. G. Georgiadis, "The problem of sharp notch in couple-stress elasticity," International Journal of Solids and Structures, vol. 48, no. 19, pp. 2630-2641, 2011.

[12] S. Itou, "Indentations of an elastic Cosserat layer by moving punches," Zeitschrift für Angewandte Mathematik und Mechanik, vol. 52, no. 2, pp. 93-99, 1972. 


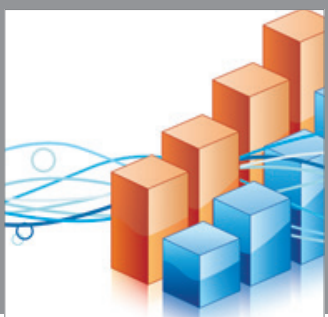

Advances in

Operations Research

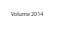

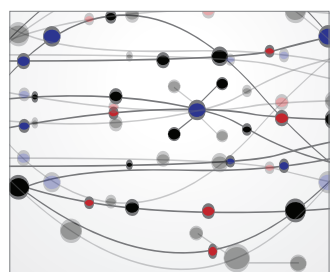

\section{The Scientific} World Journal
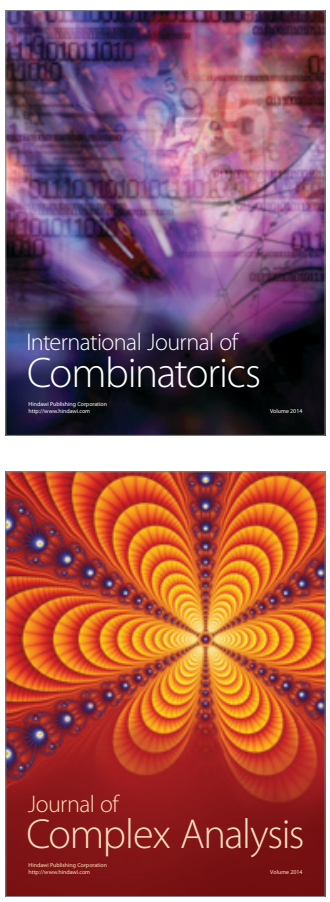

International Journal of

Mathematics and

Mathematical

Sciences
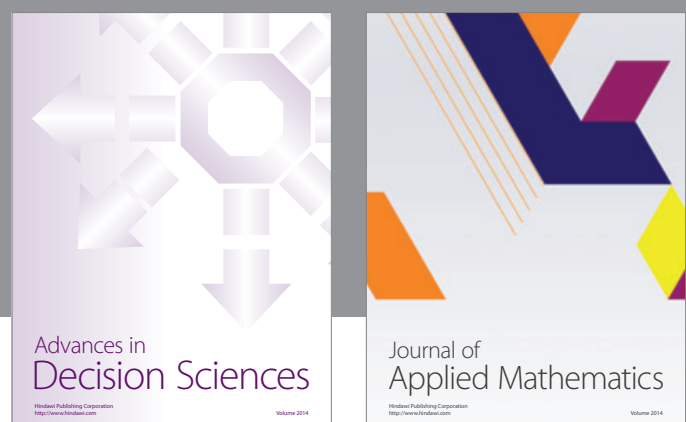

Journal of

Applied Mathematics
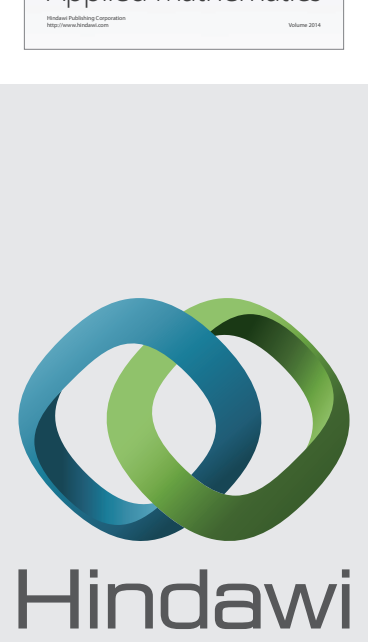

Submit your manuscripts at http://www.hindawi.com
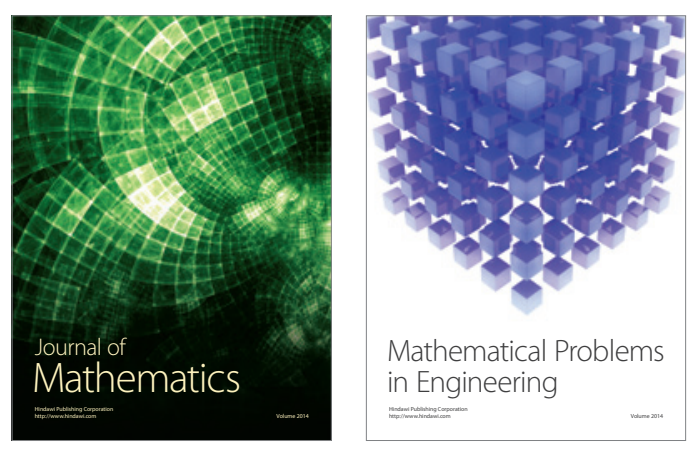

Mathematical Problems in Engineering
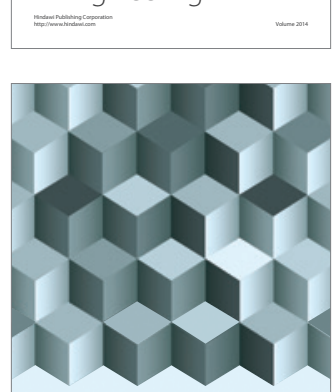

Journal of

Function Spaces
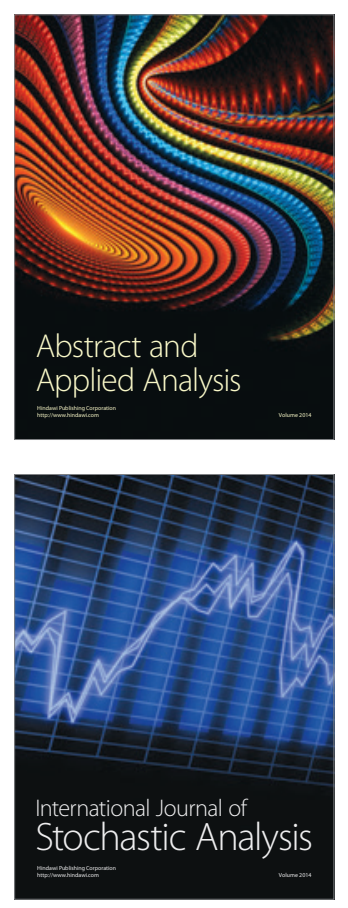

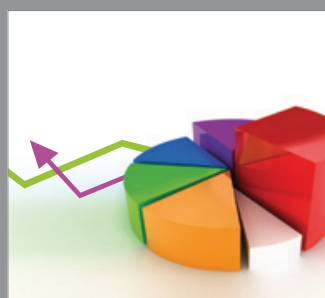

ournal of

Probability and Statistics

Promensencen
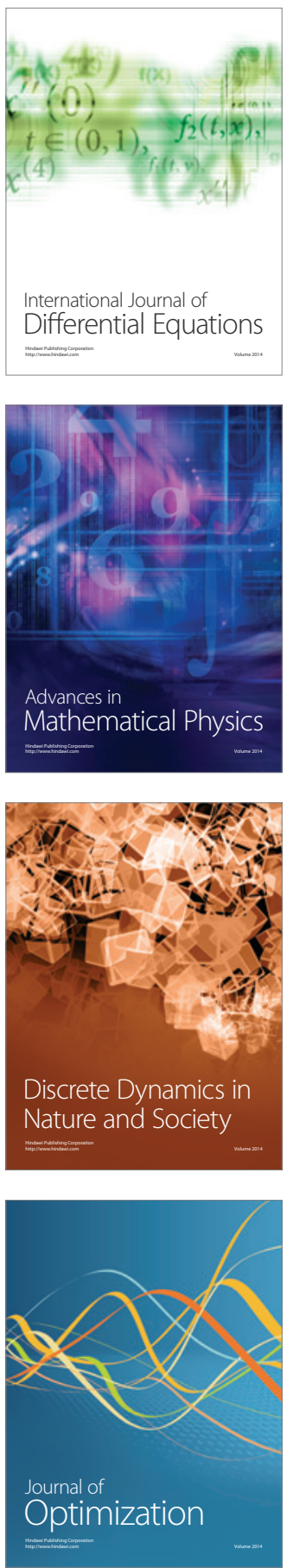